\title{
Opções atuais de transferências tendíneas para lesões posterossuperiores irreparáveis do manguito rotador*
}

\section{Current Options in Tendon Transfers for Irreparable Posterosuperior Rotator Cuff Tears}

\author{
Caio Santos Checchia ${ }^{1}$ Luciana Andrade da Silva ${ }^{1}$ Guilherme do Val Sella ${ }^{1}$ Marcelo Fregoneze ${ }^{1}$ \\ Alberto Naoki Miyazaki ${ }^{1}$ \\ ${ }^{1}$ Departamento de Ortopedia e Traumatologia, Faculdade de Ciências \\ Médicas da Santa Casa de São Paulo, São Paulo, Brasil \\ Rev Bras Ortop 2021;56(3):281-290. \begin{abstract}
Ombro e Cotovelo, Departamento de Ortopedia e Traumatologia, Faculdade de Ciências Médicas da Santa Casa de São Paulo, Rua Dr. Cesario Mota Junior, 112, Consolação, São Paulo, SP, 01221-020, Brasil
\end{abstract} \\ Endereço para correspondência Caio Santos Checchia, Grupo de \\ (e-mail: caio.checchia@gmail.com).
}

\section{Resumo \\ Palavras-chave \\ - ombro \\ - lesões do manguito rotador \\ - transferências de tendão \\ - revisão}

As grandes lesões posterossuperiores irreparáveis do manguito rotador são debilitantes e, de modo geral, requerem tratamento cirúrgico. Embora não haja consenso sobre a melhor técnica cirúrgica, as transferências tendíneas no ombro são os procedimentos mais realizados. O tendão do grande dorsal continua a ser o mais utilizado, mas diferentes modificações na técnica original têm minimizado as complicações e melhorado os resultados funcionais e a satisfação com o procedimento. Outras técnicas, como a transferência do tendão do trapézio inferior, são promissoras e devem ser consideradas, principalmente em pacientes com perda isolada da rotação externa. Este artigo é uma revisão da literatura a respeito da transferência de tendões para tratamento das lesões posterossuperiores irreparáveis do manguito rotador.

Massive irreparable posterosuperior rotator-cuff tears are debilitating lesions that usually require surgical treatment. Even though there is no consensus regarding the best surgical technique, tendinous transfers around the shoulder are the most commonly performed procedures. The latissimus dorsi tendon remains the most commonly used, but different modifications to the original technique have been shown to minimize complications and to improve functional results and satisfaction. Other techniques, such as the transfer of the lower trapezius tendon, are promising and should be considered, especially for patients with isolated loss of external rotation. The present paper is a literary review regarding tendon transfers for irreparable posterosuperior rotator-cuff tears.
Trabalho realizado no Departamento de Ortopedia e Traumatologia da Faculdade de Ciências Médicas da Santa Casa de São Paulo, Pavilhão "Fernandinho Simonsen". Diretor: professor doutor Ivan Chakkour - São Paulo (SP), Brasil. recebido

07 de Julho de 2019

aceito

10 de Janeiro de 2020

Publicado on-line

Maio 29, 2020
DOI https://doi.org/

10.1055/s-0040-1709988.

ISSN 0102-3616. (c) 2020. Sociedade Brasileira de Ortopedia e Traumatologia. All rights reserved.

This is an open access article published by Thieme under the terms of the Creative Commons Attribution-NonDerivative-NonCommercial-License, permitting copying and reproduction so long as the original work is given appropriate credit. Contents may not be used for commercial purposes, or adapted, remixed, transformed or built upon. (https://creativecommons.org/ licenses/by-nc-nd/4.0/)

Thieme Revinter Publicações Ltda., Rua do Matoso 170, Rio de Janeiro, RJ, CEP 20270-135, Brazil 


\section{Introdução}

Os reparos das lesões do manguito rotador (LMRs) estão entre as cirurgias mais comuns do ombro. ${ }^{1}$ No entanto, a cicatrização entre tendões e ossos nem sempre é bemsucedida ou previsível, ${ }^{2}$ porque depende de diversos fatores, inclusive biomecânicos e biológicos; estes últimos são influenciados pela idade do paciente. ${ }^{3} \mathrm{O}$ rápido envelhecimento da população aumentou a prevalência de fracassos nos reparos do manguito rotador. ${ }^{1,3}$ As taxas de insucesso dos reparos de lesões posterossuperiores extensas no manguito rotador variam de $21 \%$ a $91 \% \cdot{ }^{4-6}$ A prevalência de insucessos e complicações após revisões é significativamente maior ${ }^{7,8}$

Não há consenso sobre a definição de LMRs irreparáveis. Dito isto, há muitas opções terapêuticas para lesões consideradas irreparáveis, a começar pelos tratamentos não cirúrgi$\cos ^{9}$ Há controvérsia quanto à melhor opção terapêutica cirúrgica. Boileau el a ${ }^{10}$ e Walch et a ${ }^{11}$ obtiveram resultados satisfatórios após a tenotomia da cabeça longa do bíceps (CLB) isolada em pacientes idosos. Outros procedimentos foram relatados, inclusive o desbridamento do manguito rotador (com ou sem liberação concomitante do nervo supraescapular), ${ }^{12}$ reparo parcial do manguito, ${ }^{13-15}$ transferências de tendão, ${ }^{16-19}$ e artroplastia reversa de ombro (ARO). ${ }^{20}$ É provável, porém, que a ARO não seja a melhor opção em pacientes mais jovens e fisicamente ativos, porque a longevidade desses implantes nessa população ainda é desconhecida; além disso, a ARO ainda pode ser usada como procedimento de resgate após o insucesso de outras técnicas. Recentemente, a reconstrução da cápsula superior do ombro ${ }^{21-28}$ foi defendida, mas ainda há poucos resultados funcionais relatados, ${ }^{23,29}$ e um período maior de acompanhamento é necessário para confirmar sua eficácia em longo prazo. ${ }^{30}$
Apesar de ser um procedimento cirúrgico relativamente difícil, que exige uma seleção precisa dos pacientes, a transferência de tendões pode melhorar a qualidade de vida de maneira significativa. ${ }^{16,18,31}$ Isso é muito importante em pacientes jovens e fisicamente ativos com lesões classificadas de acordo com Hamada et al ${ }^{32}$ em estágios 1 e 2 (ou seja, sem artrose glenoumeral e migração estática da porção proximal da cabeça do úmero), porque a transferência pode ser o único tratamento definitivo (ou seja, em longo prazo) possível. ${ }^{30}$

O objetivo deste artigo é revisar a literatura sobre transferências de tendões em lesões posterossuperiores irreparáveis do manguito rotador (ou seja, com acometimento dos tendões supra e infraespinais), pois as LMRs mais comuns são, de longe, posterossuperiores. ${ }^{1}$ Nesse contexto, o tendão do grande dorsal (TGD) - acompanhado ou não pelo tendão do redondo maior (TRM) - é o tendão mais comumente transferido. ${ }^{33}$ As alternativas são a transferência isolada do TRM $^{34-37}$ e a transferência do tendão do trapézio inferior (TTI), que foi descrita mais recentemente. ${ }^{38,39}$

\section{Transferência do Tendão do Grande Dorsal (TCD)}

A primeira transferência, e a mais estudada, é a do TGD, originalmente descrita por Gerber et $\mathrm{al}^{17}{ }^{17}$ e realizada com uma técnica de abordagem aberta dupla (uma abordagem posterior e uma abordagem transdeltoide superior). 0 tendão transferido é fixado em sua porção superolateral à tuberosidade maior (com suturas transósseas) e anteriormente ao tendão do subescapular; assim, o grande dorsal passa a ser abdutor e rotador externo do ombro (diferentemente de seu papel original como rotador interno e adutor) ( - Figura 1). Esse procedimento é feito por dois motivos: restaurar a centralização da cabeça do úmero (uma vez que a força do subescapular anterior intacto

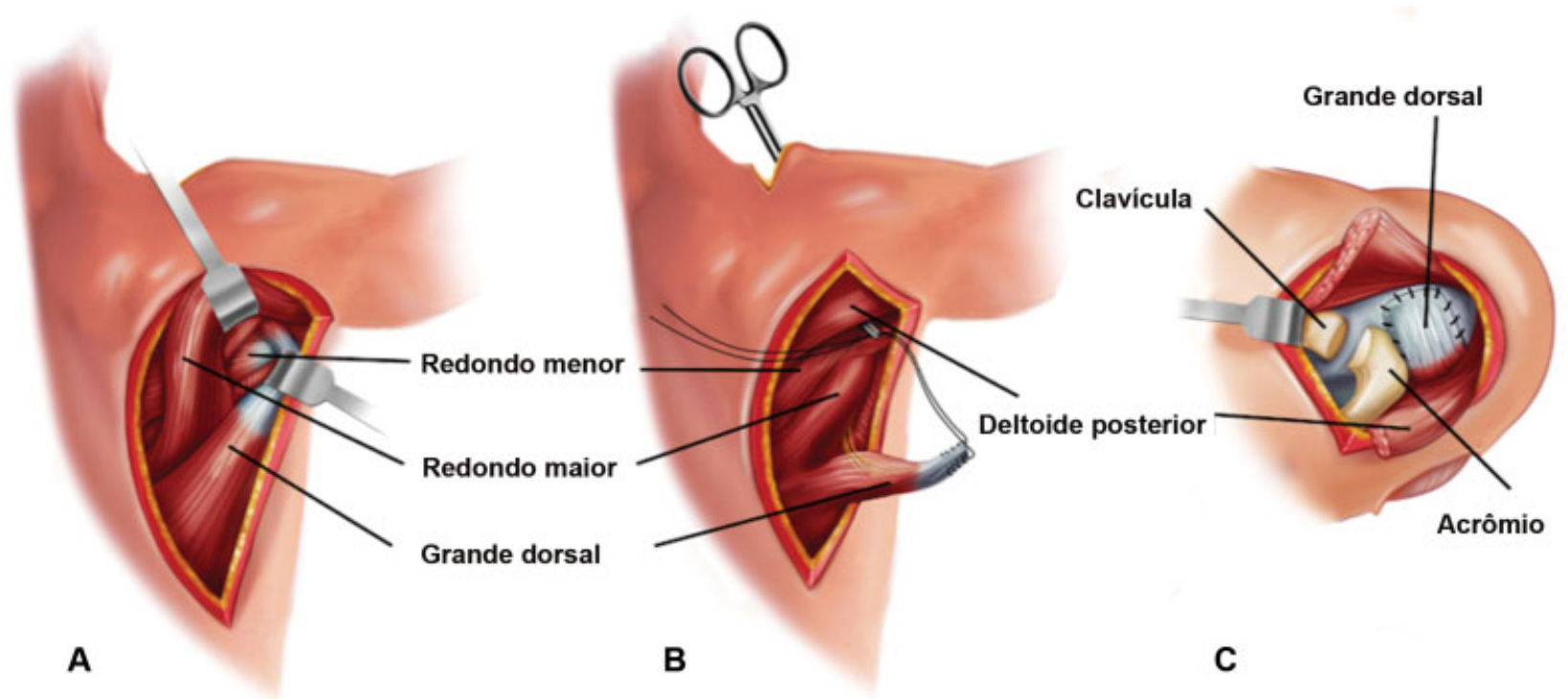

Fig. 1 (A-C) Transferência do tendão do grande dorsal pela técnica de dupla incisão. Representação do ombro direito. (A) Vista posterior. Uma incisão posterior é feita sobre a borda lateral palpável do ventre do grande dorsal, que é então separado do músculo redondo maior (que fica mais superior e mais medial ao GD). (B) Vista posterior. O tendão é desinserido do úmero e depois transportado para o espaço subacromial com uma pinça trazida da incisão superior à posterior, colocada entre o deltoide posterior e a cabeça longa do músculo tríceps. (C) Vista superior. O tendão transferido é então ancorado a uma cavidade óssea na porção superolateral da cabeça do úmero (colocação over-the-top) e qualquer manguito restante é suturado à transferência. 
agora se une à nova força posterior ${ }^{40}$ ); e melhorar a rotação externa (RE) ativa do ombro.

Gerber $^{16}$ publicou seus resultados pós-operatórios em 1992. Um total de 17 pacientes foram acompanhados por um período médio de 33 meses após a cirurgia; o autor mostrou que, no subgrupo de pacientes com função subescapular normal (12 do total de 17), houve restabelecimento de cerca de $80 \%$ da função normal do ombro. Portanto, este foi o primeiro estudo indicando que a TGD poderia ser uma alternativa segura e valiosa para esses pacientes.

Muitos outros artigos subsequentes ${ }^{18,19,31,33,41-48}$ também mostraram resultados satisfatórios após a transferência do TGD. No entanto, esses resultados satisfatórios não poderiam ser previstos com facilidade, provavelmente porque, além da técnica cirúrgica adequada, a boa seleção de pacientes também pode ser crucial, como discutido a seguir.

Embora nenhum limite superior de idade tenha sido definido, uma revisão sistemática recente ${ }^{46}$ observou que a idade média dos pacientes submetidos à transferência do TGD era de 59 anos, e mostrou que a indicação poderia ser estendida a pacientes idosos com artropatias até o estágio 3 de Hamada et al. $^{32}$

A integridade pré-operatória do deltoide e do subescapular foi considerada ${ }^{19,44,45,49}$ muito importante para a obtenção de bons resultados, pois a elevação frontal e a estabilidade do ombro, respectivamente, diminuem de forma drástica com sua insuficiência. ${ }^{16,42,50-52}$ No entanto, devemos observar que pacientes com lesões subescapulares concomitantes limitadas apenas à sua inserção proximal (mas sem disfunção subescapular, ou seja, sem sinal negativo ao teste de retirada, ou lift-off) foram tratados com sucesso desde o primeiro relato de Gerber. ${ }^{16,18,47,51}$

Outro provável fator preditivo de resultados pós-operatórios piores e diminuição da RE ativa é a atrofia préoperatória e a infiltração gordurosa (Goutallier 3 ou superior) do músculo redondo menor. ${ }^{47,52,53}$ Apesar disso, Moursy et $\mathrm{al}^{52}$ notaram que, embora pacientes com esses achados apresentassem resultados gerais piores, eram aqueles com maior ganho pós-operatório na RE; os autores concluíram que isso acontecia porque, em todos os casos, a degeneração pré-operatória do músculo redondo menor estava correlacionada à menor RE ativa antes da cirurgia. ${ }^{52}$

A boa movimentação pré-operatória do ombro também é essencial, em especial a elevação passiva acima de $80^{\circ 41}$ Além disso, ombros pseudoparéticos (definidos pela elevação ativa menor do que $90^{\circ 54}$ ) e procedimentos cirúrgicos prévios também foram correlacionados a resultados piores. ${ }^{18,55,56}$

Diferentes técnicas de transferência de TGD foram desenvolvidas. Habermeyer et $\mathrm{al}^{43}$ descreveram uma abordagem de incisão única posterior com sítio de fixação mais posterior na cabeça do úmero. Herzberg et $\mathrm{al}^{57}$ relataram uma técnica semelhante, mas, em vez disso, com fixação no sítio original de inserção do infraespinal. No entanto, os autores disseram que eventuais lesões subescapulares proximais não podem ser adequadamente tratadas por essa abordagem. Não obstante, Habermeyer et $\mathrm{al}^{43}$ mostraram resultados clínicos comparáveis aos do método de duas incisões de Gerber, ${ }^{16}$ com melhora das pontuações médias de Constant-Murley de 46,5 pontos no pré-operatório (entre 29,3 e 66,7 ) para 74,6 pontos (entre 64,5 e 81,5 ) no pós-operatório.

Há relatos recentes de transferências desse tendão por artroscopia. $^{31,48,58,59}$ Grimberg e Kany ${ }^{31}$ relataram resultados equivalentes às abordagens abertas históricas; além disso, essa abordagem artroscópica com uma incisão aumentou a resistência mecânica à tração devido à fixação do tendão em tubo com dois fios de sutura, com um total de quatro pontas de fio para fixação. ${ }^{60}$

Em 2019, Miyazaki et al $^{61}$ descreveram uma nova técnica de transferência aberta de TGD. Os autores propuseram que a técnica original tem duas desvantagens principais que podem predispor a complicações e resultados funcionais insatisfatórios: 1) ruptura pós-operatória da origem do deltoide devido à necessidade de sua desinserção do acrômio durante a abordagem superior; e 2) ruptura pósoperatória da transferência. Para evitar esses problemas, as seguintes modificações foram desenvolvidas. Por meio de uma abordagem deltopeitoral, o TGD é separado da diáfise do úmero e, então, reforçado e alongado com um enxerto tendíneo homólogo (de calcâneo ou quadricipital); por fim, o tendão é transferido em volta do úmero e fixado no aspecto superolateral da tuberosidade maior. (-Fig. 4) No entanto, nenhum resultado clínico foi publicado até o momento.

Concordamos com os achados de Moursy et $\mathrm{al}^{52}$ sobre a dificuldade de avaliação da integridade da transferência por ressonância magnética (RM). Quanto a isso, Kany et $\mathrm{al}^{62}$ publicaram em 2018 um estudo interessante. Esses autores avaliaram 60 pacientes (após a perda de 6 pacientes no acompanhamento) por um período pós-operatório médio de 35,2 meses. Em todos os casos, a técnica cirúrgica foi acompanhada pela colocação de 3 marcadores metálicos a $2 \mathrm{~cm}, 4 \mathrm{~cm}$ e $6 \mathrm{~cm}$ de distância da ponta do tendão, o que permitiu o diagnóstico fácil das rupturas de transferência com radiografias simples (-Figura 2 ). Os resultados mostraram $23(38,6 \%$ ) rupturas (-Figura 3), o que, por si só, foi um fator importante para piores resultados funcionais e satisfação pós-operatória em 7 diferentes escores funcionais e de satisfação ( $p<0,05$ em todos eles).

Checchia et al $^{30}$ levantaram a hipótese de que os resultados das transferências de TGD seriam determinados não apenas pela escolha dos pacientes ou pela insuficiência concomitante do subescapular, conforme mencionado por Gerber $^{16}$ e outros autores, ${ }^{19,44,45}$ como também respeitando cinco princípios específicos de transferência de tendões:

1. Posicionamento adequado do tendão transferido;

2. Tensão fisiológica da unidade músculo-tendínea transferida;

3. Boa fixação óssea do tendão transferido;

4. Cirurgia minimamente invasiva para redução da formação de fibrose (sem prejudicar a excursão da transferência); e

5. Transferência sinérgica.

1. O sítio ideal de fixação varia de acordo com o quadro clínico pré-operatório. De acordo com Walch et al, ${ }^{63}$ o 


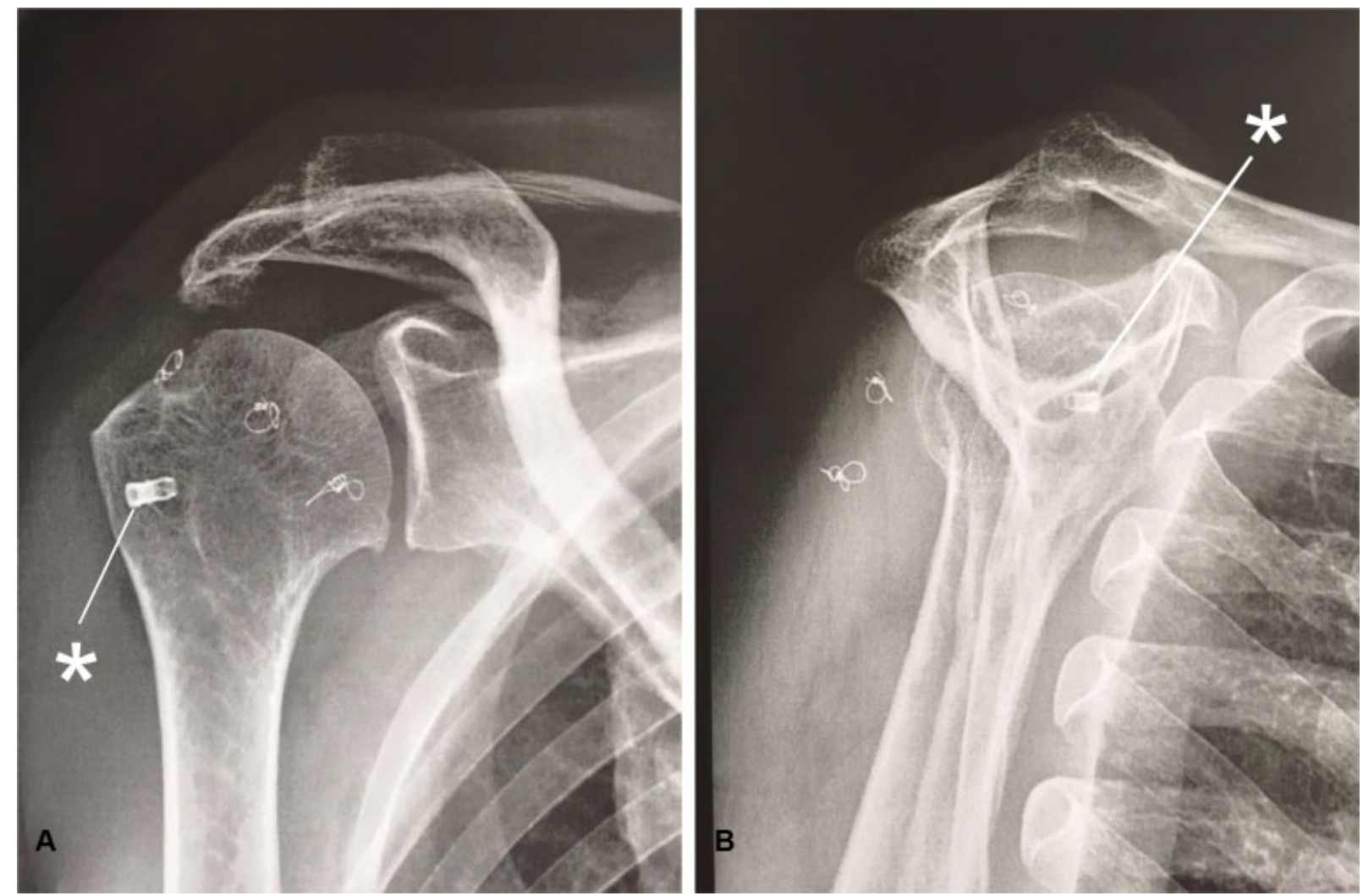

Fig. 2 (A, B) Radiografias pós-operatórias da transferência do grande dorsal com três marcadores metálicos (colocados a $2 \mathrm{~cm}$, $4 \mathrm{~cm}$ e $6 \mathrm{~cm}$ da ponta do tendão). Os asteriscos indicam a âncora metálica de um reparo anterior (sem sucesso) do manguito rotador. (A) Vista anteroposterior. (B) Vista escapular em perfil.

quadro clínico desses pacientes é variável, em especial quanto ao drop sign (também conhecido como "sinal de Hornblower" ou "sinal de trombeta"), que indica deficiência dos rotadores externos. Ombros dolorosos e pseudoparéticos com drop signs negativos (descritos por Boileau et $\mathrm{al}^{64}$ como "perda dolorosa de elevação ativa") devem ser tratados, de acordo com Gerber et al, ${ }^{17}$ com a transferência over-the-top. Isso significa que o tendão deve ser transferido acima do centro de rotação da articulação para atuar principalmente como um depressor da cabeça do úmero. Nos pacientes com drop signs isolados positivos (descritos por Boileau et $\mathrm{al}^{64}$ como "perda isolada de rotação externa"), o objetivo deve ser a fixação lateral ao centro de rotação articular. ${ }^{43,57}$

2. O segundo princípio é o controle da tensão do ventre muscular. Pode-se argumentar que a tensão insuficiente reduziria a eficácia da transferência. Alternativamente, a tensão excessiva pode danificar a unidade músculo-tendínea transferida. Foi proposto ${ }^{30}$ que, para obter tensão apropriada, antes de liberar o grande dorsal de sua fixação original, deve-se colocar dois pontos de referência no músculo enquanto o ombro está em máxima abdução e RE (que é a posição da tensão fisiológica máxima do grande dorsal). No momento da reinserção do tendão, o músculo deve ser tensionado novamente para corresponder à distância de pré-liberação entre esses dois marcadores.
3. O terceiro princípio diz respeito à resistência mecânica da fixação do tendão ao osso. Como o osso metafisário é frágil, um método de fixação resistente deve ser utilizado ${ }^{65}$ Nas abordagens abertas, acredita-se que as suturas transósseas sejam fortes o suficiente, em especial na presença de algum tipo de reforço cortical, como sugerido pelo artigo de Gerber et al. ${ }^{66}$

Nas técnicas artroscópicas, porém, implantes são necessários para boa coaptação entre o tendão e o osso. Diop et al ${ }^{67}$ publicaram um estudo biomecânico cadavérico que comparou um método de fixação padrão com âncora (em dupla-fileira simples) com a uma técnica de fixação do TGD em tubo à tuberosidade maior com um parafuso de interferência. Os autores mostraram que esta última técnica tem desempenho biomecânico maior - em termos de rigidez, deslocamentos cíclicos e carga até a falha apesar de provocar mais complicações (em especial, a fratura da tuberosidade maior durante a perfuração óssea). ${ }^{67}$ Por isso, alguns autores ${ }^{68}$ preferem a fixação do tendão em tubo com um botão cortical posicionado no sulco bicipital, evitando a perfuração da tuberosidade maior.

Em outra técnica que melhora a cicatrização entre o tendão e o osso, fragmentos de osso cortical são removidos com o TGD (por meio de uma delicada osteotomia superficial do córtex umeral, e não uma simples tenotomia). De fato, Moursy et $a^{52}$ mostraram que essa 

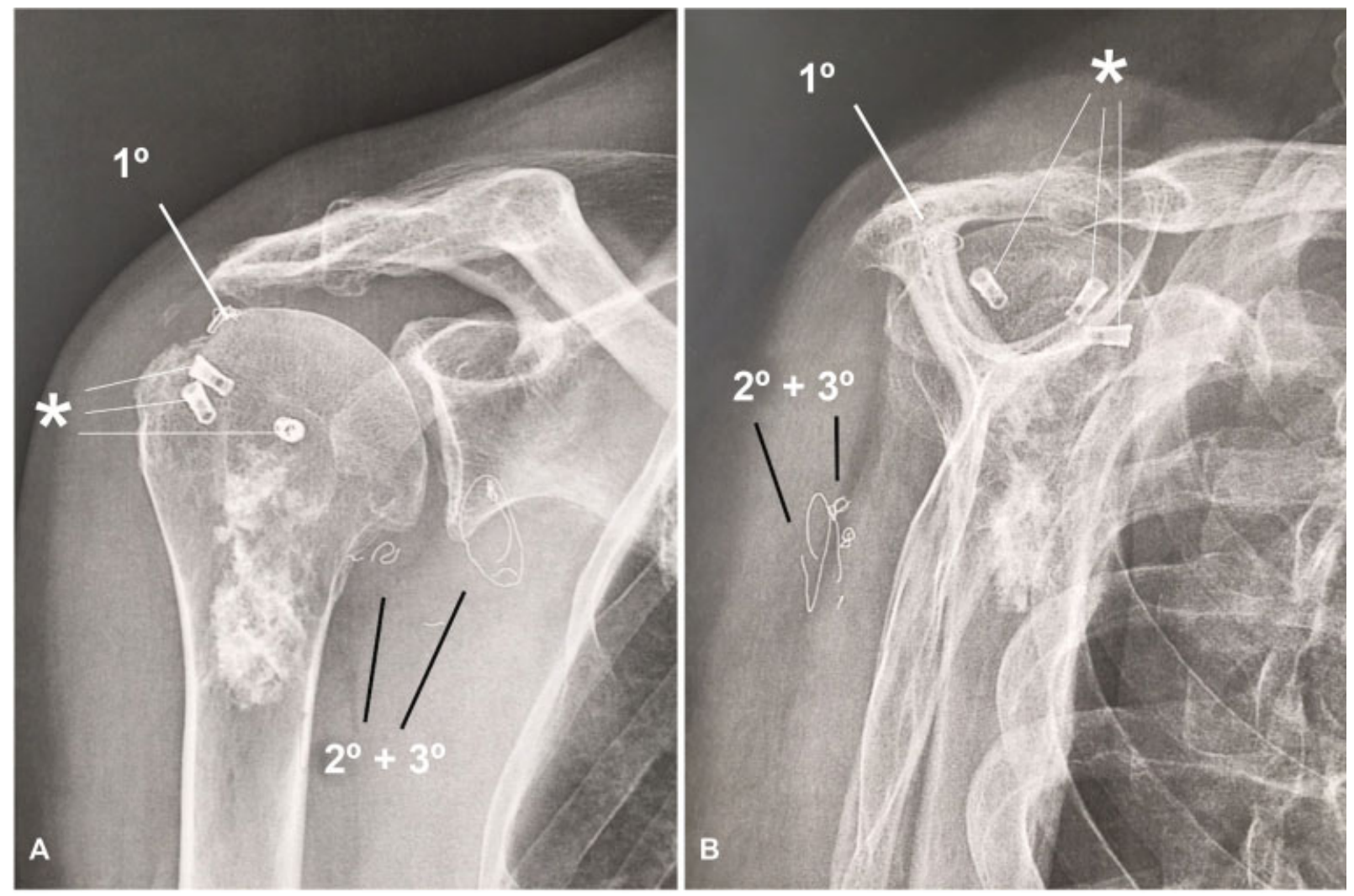

Fig. 3 (A, B) Ruptura pós-operatória da transferência do tendão do grande dorsal (TGD). Observe a maior distância entre o primeiro marcador intacto $\left(1^{\circ}\right)$ e o segundo e terceiro $\left(2^{\circ}\right.$ e $\left.3^{\circ}\right)$ marcadores metálicos deslocados. Os asteriscos indicam âncoras metálicas de um reparo prévio (sem sucesso) do manguito rotador. Observe que a lesão calcificada na parte proximal do úmero é um encondroma, não relacionado ao procedimento ou à ruptura do manguito rotador. (A) Vista anteroposterior. (B) Vista escapular em perfil.

modificação reduz estatisticamente o número de rupturas pós-operatórias e gera melhores resultados funcionais. Uma alternativa já mencionada é o reforço tendíneo com um aloenxerto tendinoso, como proposto por Miyazaki et al. $^{61}$ (-Fig. 4 )

4. O quarto princípio visa limitar o trauma cirúrgico aos tecidos. Pelo que sabemos, esse princípio não é apoiado em nenhum dado empírico, à exceção dos achados de Warner e Parsons, ${ }^{56}$ que obtiveram resultados abaixo do ideal em casos de revisões ou de múltiplas transferências simultâneas de tendões. No entanto, podemos argumentar que a menor quantidade de trauma pode melhorar a cicatrização, pois minimiza a desvascularização iatrogênica e permite maior excursão tendínea devido à menor geração de cicatrizes nos tecidos moles circundantes. Além disso, técnicas minimamente invasivas, como abordagens artroscópicas, preservam o ventre do músculo deltoide, que é muito importante para a função do ombro em caso de deficiência do manguito rotador. Mesmo que todas as etapas da transferência de TGD possam ser executadas exclusivamente por meio de uma abordagem artroscópica (Lafosse, Nice Shoulder Course, 2016, dados não publicados), acreditamos que isso deva ser evitado, uma vez que nossa experiência demonstrou que problemas na liberação do grande dorsal do ápice da escápula (que, em nossa opinião, não pode ser realizada por artroscopia) leva ao desenvolvimento de escápula alada no período pós-operatório.
5. Também é possível argumentar que a transferência de TGD não respeita o quinto princípio da transferência tendínea, porque esse músculo não é, a princípio, um agonista da elevação e da RE, e sua linha de ação é mais inferior e posterior em relação aos tendões originais do manguito (-Figura 5). Talvez este seja o principal motivo dos resultados eletromiográficos controversos e imperfeitos, ${ }^{43,47,69}$ assim como de alguns relatos de resultados clínicos insatisfatórios. $^{18,70}$

\section{Transferência do Tendão do Redondo Maior (TRM)}

Há pouquíssimos estudos publicados acerca da transferência isolada do TRM nas LMRs irreparáveis. Todos foram realizados em pacientes com deficiência isolada do infraespinal. A primeira série foi relatada em 1998 por Celli et al, ${ }^{35}$ que alcançaram bons resultados funcionais e satisfação em todos os seus 6 pacientes. Recentemente, Celli et al, ${ }^{34}$ publicaram os resultados em longo prazo de vinte pacientes com manutenção da melhora dos escores de Constant-Murley no período pós-operatório.

Recentemente, Henseler et $\mathrm{al}^{36}$ publicaram os resultados em curto prazo da transferência de TRM. Em dois anos de acompanhamento, os pacientes apresentaram melhoras significativas $(p<0,05)$ na RE, na elevação, na escala visual analógica, e nos escores de Constant-Murley. No acompanhamento em médio prazo (média de seis anos), Mansat et $\mathrm{al}^{37}$ avaliaram 

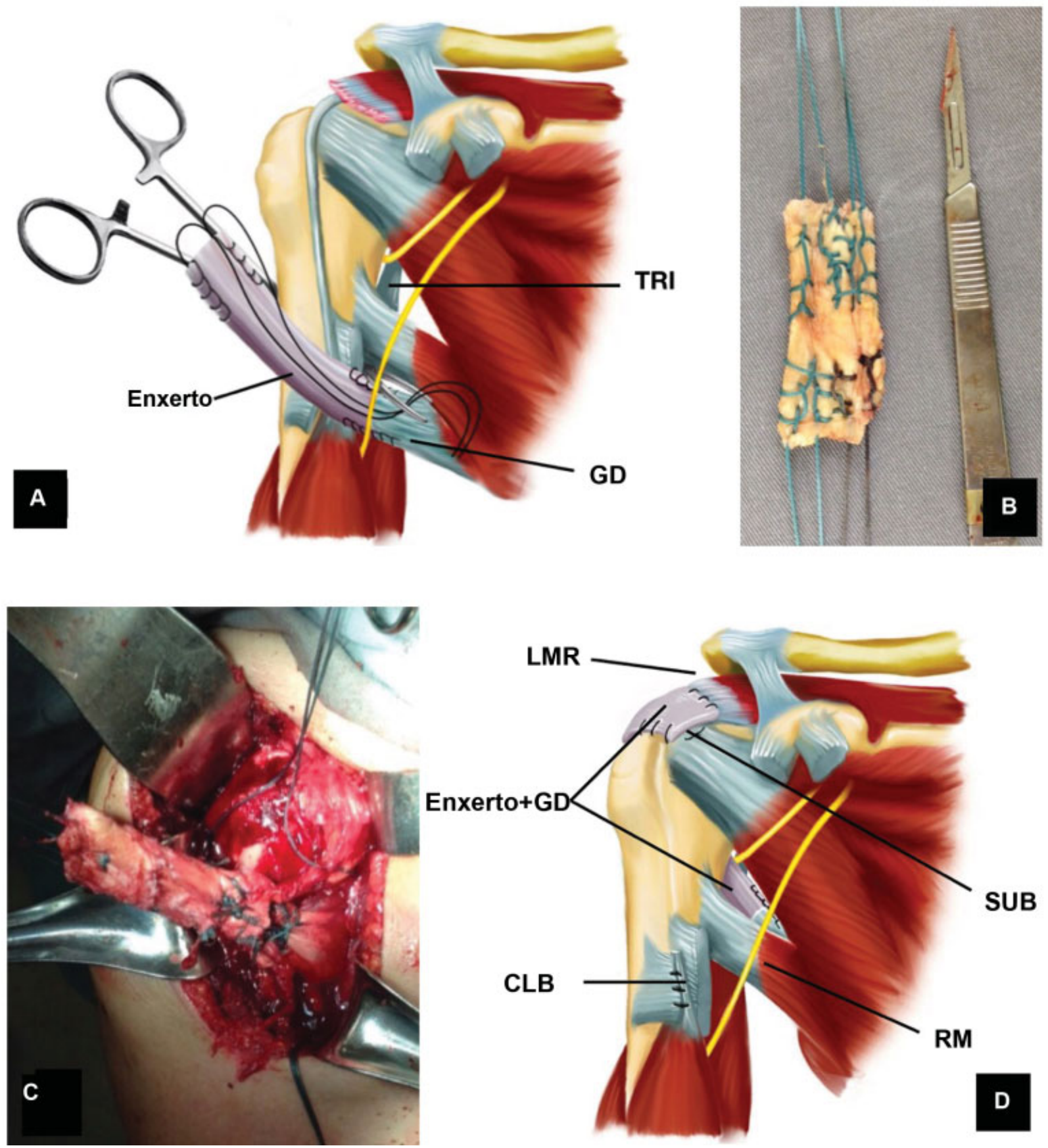

Fig. 4 (A-D) Transferência do tendão do grande dorsal (TGD) alongado e reforçado com um enxerto tendinoso homólogo realizada por uma abordagem deltopeitoral isolada, conforme descrito por Miyazaki et al. ${ }^{61}$ (A) A figura mostra o preparo do tendão antes da transferência. (B) Preparo do enxerto. (C) Depois da passagem da transferência atrás do úmero. (D) A figura representa a configuração final do TGD. Abreviaturas: TRI, tríceps; GD. grande dorsal; LMR, lesão do manguito rotador; CLB, cabeça longa do bíceps; RM, redondo maior; SUB, subescapular.

os resultados de transferências de TRM, que foram similares aos de Henseler et al. ${ }^{36}$ É interessante notar que os autores ${ }^{37}$ puderam identificar fatores prognósticos pré-operatórios negativos, como cirurgias prévias e LMRs envolvendo o subescapular, além de dois fatores prognósticos positivos: o acometimento isolado do infraespinal e a presença de um redondo menor funcional. Além disso, Mansat et $\mathrm{al}^{37}$ descreveram as seguintes recomendações para a transferência de TRM: os pacientes devem ter menos de 55 anos de idade e entendimento adequado da lesão e do tratamento, além de subescapular e cabo supraespinal anterior intactos.

\section{Transferência do Tendão do Trapézio Inferior (TTI)}

A incisão dupla (uma técnica de transferência de TTI prolongada com enxerto tendinoso homólogo ou autólogo) foi recentemente investigada como uma alternativa à transferência de TGD em LMRs posteriores irreparáveis e lesões musculotendíneas isoladas crônicas do infraespinal. ${ }^{33,38,39,71}$ Além da provável maior facilidade técnica em comparação ao TGD, a linha de tração das fibras musculares do trapézio inferior reproduz melhor a linha do infraespinal (-Figura 5). Também 

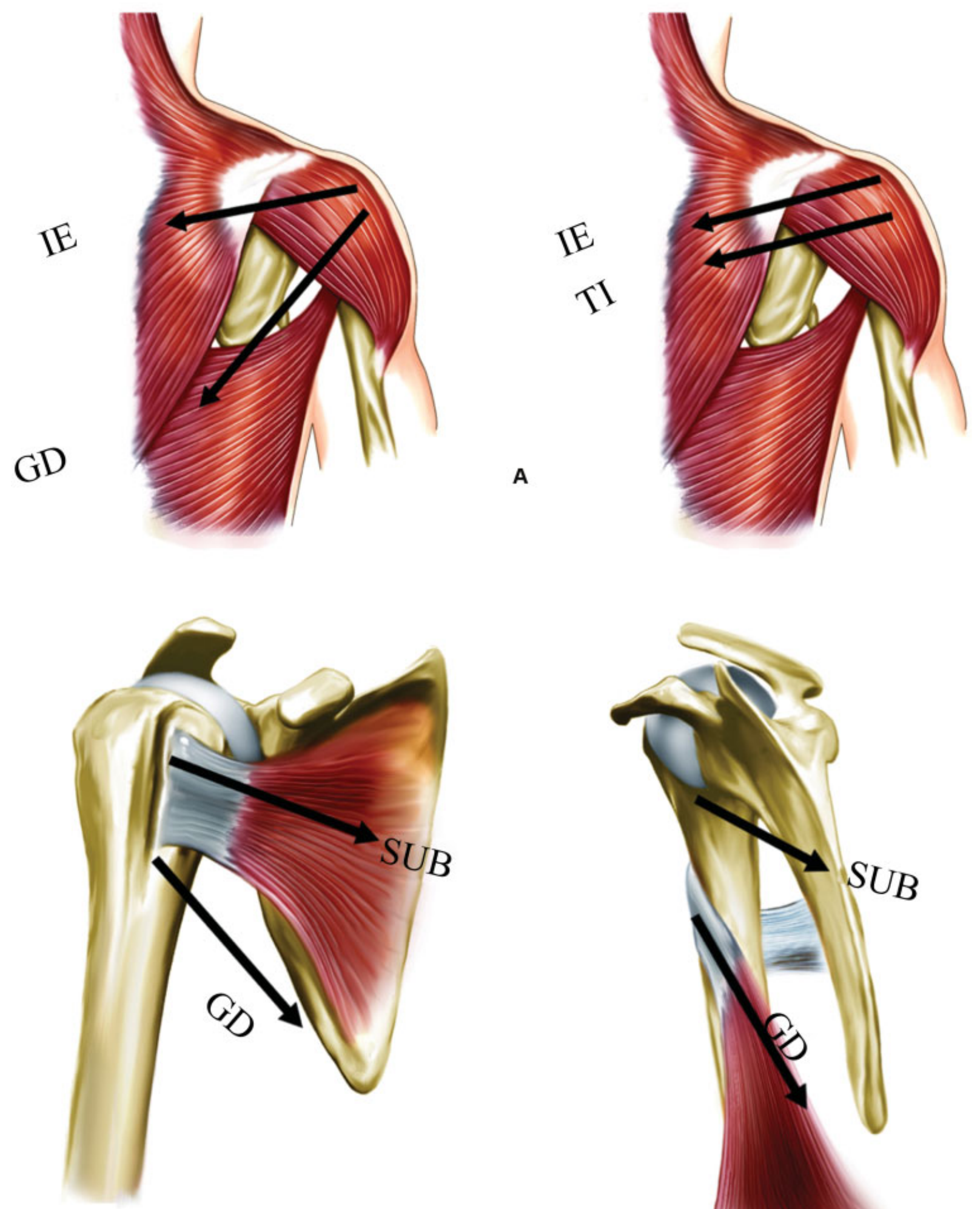

B

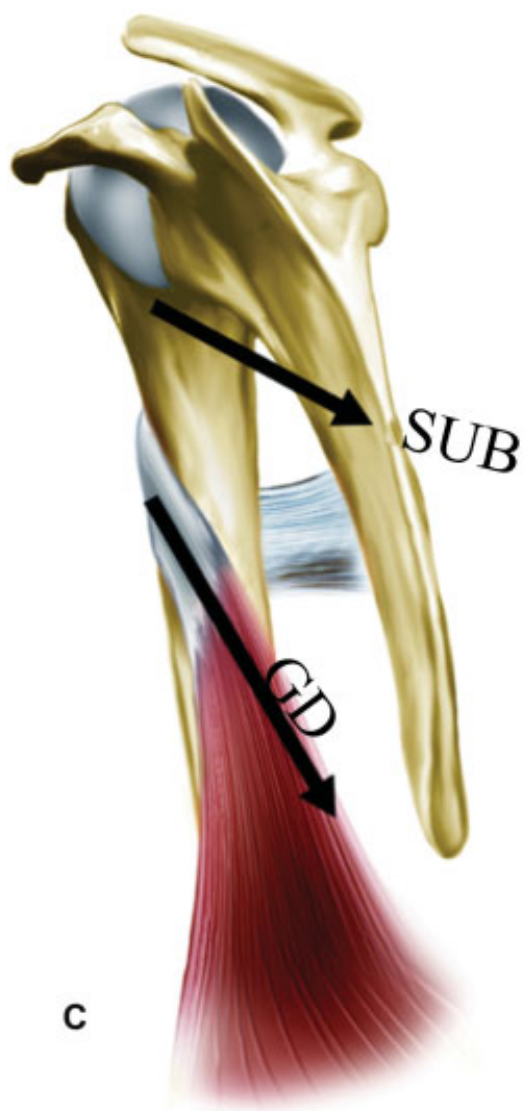

Fig. 5 (A-C) Desenhos do ombro direito. A linha de tração do trapézio inferior (TI) replica melhor a linha do infraespinal (IE) do que aquela gerada pelo grande dorsal (GD). A linha de tração do GD replica melhor a linha de tração do subescapular (SUB). (A) Vista posterior. (B) Vista anterior. (C) Vista medial. 
foi demonstrado que as forças de tensão e excursão do trapézio são muito semelhantes às do infraespinal. ${ }^{38,39}$

Em um estudo com cadáveres, Omid et $\mathrm{al}^{39}$ concluíram que a transferência de TTI era biomecanicamente superior à de TGD, com maiores forças de RE. Hartzler et $\mathrm{al}^{38}$ também observaram a melhora da RE com o braço ao lado em comparação à transferência de TGD, mas a transferência de TGD foi melhor no restauro da elevação dianteira e da RE a $90^{\circ}$ de abdução. Recentemente, em 2019, Reddy et $\mathrm{al}^{72}$ mostraram, por meio de um estudo biomecânico virtual em três dimensões (3D) (com execução virtual de transferência de TGD e TTI em um software), que o TTI proporcionou melhores vetores de abdução e RE quando transferido para a inserção do infraespinal. No entanto, o desempenho da transferência de TGD foi superior quando realizada na inserção supraespinal. De modo geral, a transferência de TTI teve uma vantagem biomecânica em comparação à de TGD devido a maiores vetores de abdução.

Em 2016, Elhassan et al, ${ }^{71}$ foram os primeiros a relatar os resultados funcionais dessa técnica. Os autores avaliaram 33 pacientes ( 26 homens com média de 53 anos de idade; faixa de 31 a 66 anos) com acompanhamento médio de 47 meses (faixa de 24 a 73 meses). À exceção de um paciente com um índice de massa corpórea de $36 \mathrm{~kg} / \mathrm{m}^{2}$, todos apresentaram melhoras estatisticamente significativas em pontuações de dor, Subjective Shoulder Value (SSV) e Disabilities of the Arm, Shoulder and Hand (DASH), além de melhora estatisticamente significativa em todos os movimentos ativos do ombro. Seus resultados foram, portanto, os primeiros a mostrar que esse tipo de tratamento pode ser uma boa alternativa, pelo menos no acompanhamento em curto e médio prazos.

Em 2016, Elhassan et $\mathrm{al}^{73}$ descreveram uma modificação em sua técnica original, em que, em vez de criar uma segunda abordagem lateral aberta, a transferência era fixada na tuberosidade maior sob visualização artroscópica. No entanto, os autores não publicaram os resultados desta modificação. No entanto, em 2018, Valenti e Werthel ${ }^{74}$ publicaram os resultados seguindo quase a mesma técnica publicada por Elhassan et al; $;^{73}$ a única diferença foi o uso de um enxerto autólogo de tendão semitendíneo em vez de um enxerto homólogo de tendão calcâneo. Os autores avaliaram 14 pacientes por um período médio de 24 meses (faixa de 12 a 36 meses). Seus resultados mostraram ganho na RE com o braço ao lado em $24^{\circ}$ e, em $90^{\circ}$ de abdução, de $40^{\circ}$. O sinal lag e o sinal de Hornblower desapareceram em todos os pacientes que os apresentavam antes da cirurgia. 0 escore de Constant-Murley melhorou de 35 (no pré-operatório) para 60 pontos (no pós-operatório), o Simple Shoulder Test (SST) passou de 3,5 para 7,5, o SSV, de 30 a $60 \%$ e a dor diminuiu de 7 para 2 (escala visual analógica, EVA). Houve dois casos de hematomas e uma revisão em decorrência de infecção.

Não temos conhecimento de nenhum outro estudo publicado que mostre os resultados da transferência de TTI em casos de LMRs irreparáveis. Apesar de tudo isso, até agora não existem estudos clínicos que sugiram a superioridade da transferência do TGD ou do TTI.

\section{Conclusão}

As LMRs posterossuperiores irreparáveis podem ser debilitantes, e o tratamento de re-rupturas do manguito rotador ainda é difícil. Diferentes técnicas foram desenvolvidas, e os benefícios propostos da transferência de tendões são o alívio da dor e o aumento da amplitude de movimento e da força do ombro. A transferência de TGD continua a ser o método mais utilizado, e diferentes modificações na técnica original minimizaram as complicações e melhoraram os resultados funcionais e a satisfação. No entanto, as transferências de TTI são promissoras, e devem ser consideradas, principalmente em pacientes com perda isolada de RE. Seus resultados, no entanto, são limitados a duas séries de casos, com relato somente do acompanhamento em curto e médio prazos.

\section{Conflito de Interesses}

Os autores declaram não haver conflito de interesses.

\section{Referências}

1 Tashjian RZ. Epidemiology, natural history, and indications for treatment of rotator cuff tears. Clin Sports Med 2012;31(04):589-604

2 Galatz LM, Ball CM, Teefey SA, Middleton WD, Yamaguchi K. The outcome and repair integrity of completely arthroscopically repaired large and massive rotator cuff tears. J Bone Joint Surg Am 2004;86(02):219-224

3 Longo UG, Berton A, Papapietro N, Maffulli N, Denaro V. Epidemiology, genetics and biological factors of rotator cuff tears. Med Sport Sci 2012;57:1-9

4 Bartl C, Kouloumentas P, Holzapfel K, et al. Long-term outcome and structural integrity following open repair of massive rotator cuff tears. Int J Shoulder Surg 2012;6(01):1-8

5 Jo $\mathrm{CH}$, Shin JS, Lee YG, et al. Platelet-rich plasma for arthroscopic repair of large to massive rotator cuff tears: a randomized, singleblind, parallel-group trial. Am J Sports Med 2013;41(10):2240-2248

6 Kim SJ, Kim SH, Lee SK, Seo JW, Chun YM. Arthroscopic repair of massive contracted rotator cuff tears: aggressive release with anterior and posterior interval slides do not improve cuff healing and integrity. J Bone Joint Surg Am 2013;95(16):1482-1488

7 Shamsudin A, Lam PH, Peters K, Rubenis I, Hackett L, Murrell GA. Revision versus primary arthroscopic rotator cuff repair: a 2-year analysis of outcomes in 360 patients. Am J Sports Med 2015;43 (03):557-564

8 Parnes N, DeFranco M, Wells JH, Higgins LD, Warner JJ. Complications after arthroscopic revision rotator cuff repair. Arthroscopy 2013;29(09):1479-1486

9 Bokor DJ, Hawkins RJ, Huckell GH, Angelo RL, Schickendantz MS. Results of nonoperative management of full-thickness tears of the rotator cuff. Clin Orthop Relat Res 1993;(294):103-110

10 Boileau P, Baqué F, Valerio L, Ahrens P, Chuinard C, Trojani C. Isolated arthroscopic biceps tenotomy or tenodesis improves symptoms in patients with massive irreparable rotator cuff tears. J Bone Joint Surg Am 2007;89(04):747-757

11 Walch G, Edwards TB, Boulahia A, Nové-Josserand L, Neyton L, Szabo I. Arthroscopic tenotomy of the long head of the biceps in the treatment of rotator cuff tears: clinical and radiographic results of 307 cases. J Shoulder Elbow Surg 2005;14(03):238-246

12 Gartsman GM. Massive, irreparable tears of the rotator cuff Results of operative debridement and subacromial decompression. J Bone Joint Surg Am 1997;79(05):715-721

13 Kim SJ, Lee IS, Kim SH, Lee WY, Chun YM. Arthroscopic partial repair of irreparable large to massive rotator cuff tears. Arthroscopy 2012;28(06):761-768 
14 Iagulli ND, Field LD, Hobgood ER, Ramsey JR, Savoie FH 3rd. Comparison of partial versus complete arthroscopic repair of massive rotator cuff tears. Am J Sports Med 2012;40(05): 1022-1026

15 Berth A, Neumann W, Awiszus F, Pap G. Massive rotator cuff tears: functional outcome after debridement or arthroscopic partial repair. J Orthop Traumatol 2010;11(01):13-20

16 Gerber C. Latissimus dorsi transfer for the treatment of irreparable tears of the rotator cuff. Clin Orthop Relat Res 1992;(275): $152-160$

17 Gerber C, Vinh TS, Hertel R, Hess CW. Latissimus dorsi transfer for the treatment of massive tears of the rotator cuff. A preliminary report. Clin Orthop Relat Res 1988;(232):51-61

18 Iannotti JP, Hennigan S, Herzog R, et al. Latissimus dorsi tendon transfer for irreparable posterosuperior rotator cuff tears. Factors affecting outcome. J Bone Joint Surg Am 2006;88(02):342-348

19 Warner JJ. Management of massive irreparable rotator cuff tears: the role of tendon transfer. Instr Course Lect 2001;50:63-71

20 Mulieri P, Dunning P, Klein S, Pupello D, Frankle M. Reverse shoulder arthroplasty for the treatment of irreparable rotator cuff tear without glenohumeral arthritis. J Bone Joint Surg Am 2010;92(15):2544-2556

21 Mihata T, Watanabe C, Fukunishi K, Ohue M, Tsujimura T, Kinoshita M. Arthroscopic Superior Capsular Reconstruction Restores Shoulder Stability and Function in Patients with Irreparable Rotator Cuff Tears: A Prospective Study (SS-15). Arthroscopy 2011;27(05):e36-e37

22 Mihata T, McGarry MH, Pirolo JM, Kinoshita M, Lee TQ. Superior capsule reconstruction to restore superior stability in irreparable rotator cuff tears: a biomechanical cadaveric study. Am J Sports Med 2012;40(10):2248-2255

23 Mihata T, Lee TQ Watanabe C, et al. Clinical results of arthroscopic superior capsule reconstruction for irreparable rotator cuff tears. Arthroscopy 2013;29(03):459-470

24 Gracitelli ME, Beraldo RA, Malavolta EA, Assunção JH, de Oliveira DR, Ferreira Neto AA. Reconstrução da cápsula superior com aloenxerto de fáscia lata para roturas irreparáveis do tendão do músculo supraespinhal. Rev Bras Ortop 2019;54(05):591-596

25 Burkhart SS, Denard PJ, Adams CR, Brady PC, Hartzler RU. Arthroscopic Superior Capsular Reconstruction for Massive Irreparable Rotator Cuff Repair. Arthrosc Tech 2016;5(06):e1407-e1418

26 Hartzler RU, Burkhart SS. Superior Capsular Reconstruction. Orthopedics 2017;40(05):271-280

27 Hirahara AM, Adams CR. Arthroscopic Superior Capsular Reconstruction for Treatment of Massive Irreparable Rotator Cuff Tears. Arthrosc Tech 2015;4(06):e637-e641

28 Tokish JM, Beicker C. Superior Capsule Reconstruction Technique Using an Acellular Dermal Allograft. Arthrosc Tech 2015;4(06): e833-e839

29 Denard PJ, Brady PC, Adams CR, Tokish JM, Burkhart SS. Preliminary Results of Arthroscopic Superior Capsule Reconstruction with Dermal Allograft. Arthroscopy 2018;34(01):93-99

30 Checchia C, Domos P, Grimberg J, Kany J. Current Options in Tendon Transfers for Irreparable Rotator Cuff Tears. JBJS Rev 2019; 7(02): 6

31 Grimberg J, Kany J. Latissimus dorsi tendon transfer for irreparable postero-superior cuff tears: current concepts, indications, and recent advances. Curr Rev Musculoskelet Med 2014;7(01):22-32

32 Hamada K, Fukuda H, Mikasa M, Kobayashi Y. Roentgenographic findings in massive rotator cuff tears. A long-term observation. Clin Orthop Relat Res 1990;(254):92-96

33 Omid R, Lee B. Tendon transfers for irreparable rotator cuff tears. J Am Acad Orthop Surg 2013;21(08):492-501

34 Celli A, Marongiu MC, Rovesta C, Celli L. Transplant of the teres major in the treatment of irreparable injuries of the rotator cuff (long-term analysis of results). Chir Organi Mov 2005;90(02): $121-132$
35 Celli L, Rovesta C, Marongiu MC, Manzieri S. Transplantation of teres major muscle for infraspinatus muscle in irreparable rotator cuff tears. J Shoulder Elbow Surg 1998;7(05):485-490

36 Henseler JF, Nagels J, van der Zwaal P, Nelissen RGHH. Teres major tendon transfer for patients with massive irreparable posterosuperior rotator cuff tears: Short-term clinical results. Bone Joint J 2013;95-B(04):523-529

37 Mansat P, Dotziz A, Bellumore Y, Mansat M. Teres major flap: surgical anatomy, technique of harvesting, methods of fixation, postoperative management. In: Tendon transfer for irreparable cuff tear [Internet]. SpringerParis2011 [cited 2017 Sep 23]. p. 49-64. (Collection GECO). Available from: https://link.springer.com/chapter/10.1007/978-2-8178-0049-3_5

38 Hartzler RU, Barlow JD, An KN, Elhassan BT. Biomechanical effectiveness of different types of tendon transfers to the shoulder for external rotation. J Shoulder Elbow Surg 2012;21(10): 1370-1376

39 Omid R, Heckmann N, Wang L, McGarry MH, Vangsness CT Jr, Lee TQ. Biomechanical comparison between the trapezius transfer and latissimus transfer for irreparable posterosuperior rotator cuff tears. J Shoulder Elbow Surg 2015;24(10):1635-1643

40 Burkhart SS, Athanasiou KA, Wirth MA. Margin convergence: a method of reducing strain in massive rotator cuff tears. Arthroscopy 1996;12(03):335-338

41 Buchmann S, Plath JE, Imhoff AB. [Latissimus dorsi transfer for the treatment of irreparable rotator tears: indication, surgical technique, and modifications]. Oper Orthop Traumatol 2012;24(06): 502-512

42 El-Azab HM, Rott O, Irlenbusch U. Long-term follow-up after latissimus dorsi transfer for irreparable posterosuperior rotator cuff tears. J Bone Joint Surg Am 2015;97(06):462-469

43 Habermeyer P, Magosch P, Rudolph T, Lichtenberg S, Liem D. Transfer of the tendon of latissimus dorsi for the treatment of massive tears of the rotator cuff: a new single-incision technique. J Bone Joint Surg Br 2006;88(02):208-212

44 Irlenbusch U, Bracht M, Gansen HK, Lorenz U, Thiel J. Latissimus dorsi transfer for irreparable rotator cuff tears: a longitudinal study. J Shoulder Elbow Surg 2008;17(04):527-534

45 Miniaci A, MacLeod M. Transfer of the latissimus dorsi muscle after failed repair of a massive tear of the rotator cuff. A two to five-year review. J Bone Joint Surg Am 1999;81(08):1120-1127

46 Namdari S, Voleti P, Baldwin K, Glaser D, Huffman GR. Latissimus dorsi tendon transfer for irreparable rotator cuff tears: a systematic review. J Bone Joint Surg Am 2012;94(10):891-898

47 Nové-Josserand L, Costa P, Liotard JP, Safar JF, Walch G, Zilber S. Results of latissimus dorsi tendon transfer for irreparable cuff tears. Orthop Traumatol Surg Res 2009;95(02):108-113

48 Villacis D, Merriman J, Wong K, Rick Hatch GF III. Latissimus dorsi tendon transfer for irreparable rotator cuff tears: a modified technique using arthroscopy. Arthrosc Tech 2013;2(01):e27-e30

49 Irlenbusch U, Bensdorf M, Gansen HK, Lorenz U. [Latissimus dorsi transfer in case of irreparable rotator cuff tear-a comparative analysis of primary and failed rotator cuff surgery, in dependence of deficiency grade and additional lesions]. Z Orthop Ihre Grenzgeb 2003;141(06):650-656

50 Glanzmann MC, Goldhahn J, Flury M, Schwyzer HK, Simmen BR. Deltoid flap reconstruction for massive rotator cuff tears: midand long-term functional and structural results. J Shoulder Elbow Surg 2010;19(03):439-445

51 Werner CML, Zingg PO, Lie D, Jacob HAC, Gerber C. The biomechanical role of the subscapularis in latissimus dorsi transfer for the treatment of irreparable rotator cuff tears. J Shoulder Elbow Surg 2006;15(06):736-742

52 Moursy M, Forstner R, Koller H, Resch H, Tauber M. Latissimus dorsi tendon transfer for irreparable rotator cuff tears: a modified technique to improve tendon transfer integrity. J Bone Joint Surg Am 2009;91(08):1924-1931 
53 Costouros JG, Espinosa N, Schmid MR, Gerber C. Teres minor integrity predicts outcome of latissimus dorsi tendon transfer for irreparable rotator cuff tears. J Shoulder Elbow Surg 2007;16(06):727-734

54 Tokish JM, Alexander TC, Kissenberth MJ, Hawkins RJ. Pseudoparalysis: a systematic review of term definitions, treatment approaches, and outcomes of management techniques. J Shoulder Elbow Surg 2017;26(06):e177-e187

55 Gerber C, Maquieira G, Espinosa N. Transfert du grand dorsal pour le traitement des ruptures massives de la coiffe des rotateurs: quels facteurs déterminent le résultat final? Rev Chir Orthop Repar Appar Mot 2004;90(05, Suppl 1):158-162

56 Warner JJ, Parsons IM IV. Latissimus dorsi tendon transfer: a comparative analysis of primary and salvage reconstruction of massive, irreparable rotator cuff tears. J Shoulder Elbow Surg 2001;10(06):514-521

57 Herzberg G, Urien JP, Dimnet J. Potential excursion and relative tension of muscles in the shoulder girdle: relevance to tendon transfers. J Shoulder Elbow Surg 1999;8(05):430-437

58 Castricini R, Longo UG, De Benedetto M, et al. ArthroscopicAssisted Latissimus Dorsi Transfer for the Management of Irreparable Rotator Cuff Tears: Short-Term Results. J Bone Joint Surg Am 2014;96(14):e119

59 Grimberg J, Kany J, Valenti P, Amaravathi R, Ramalingam AT. Arthroscopic-assisted latissimus dorsi tendon transfer for irreparable posterosuperior cuff tears. Arthroscopy 2015;31(04):599-607.e1

60 Sirveaux F, Favard L, Oudet D, Huquet D, Walch G, Molé D. Grammont inverted total shoulder arthroplasty in the treatment of glenohumeral osteoarthritis with massive rupture of the cuff. Results of a multicentre study of 80 shoulders. J Bone Joint Surg $\mathrm{Br}$ 2004;86(03):388-395

61 Miyazaki AN, Checchia CS, Lopes WC, Fonseca Filho JM, do Val Sella G, Silva LA. Transferência tendínea do grande dorsal com enxerto tendíneo homólogo para as lesões irreparáveis do manguito rotador: técnica cirúrgica. Rev Bras Ortop 2019;54(01):99-103

62 Kany J, Grimberg J, Amaravathi RS, Sekaran P, Scorpie D, Werthel JD. Arthroscopically-Assisted Latissimus Dorsi Transfer for Irreparable Rotator Cuff Insufficiency: Modes of Failure and Clinical Correlation. Arthroscopy 2018;34(04):1139-1150

63 Walch G, Boulahia A, Calderone S, Robinson AH. The 'dropping' and 'hornblower's' signs in evaluation of rotator-cuff tears. J Bone Joint Surg Br 1998;80(04):624-628
64 Boileau P, Chuinard C, Roussanne Y, Neyton L, Trojani C. Modified latissimus dorsi and teres major transfer through a single deltopectoral approach for external rotation deficit of the shoulder: as an isolated procedure or with a reverse arthroplasty. J Shoulder Elbow Surg 2007;16(06):671-682

65 Clavert P, Bouchaïb J, Sommaire C, Flurin PH, Hardy P. Observe-ton une modification de la densité osseuse du tubercule majeur après 70ans? Rev Chir Orthop Traumatol 2014;100(01):94-96

66 Gerber C, Schneeberger AG, Beck M, Schlegel U. Mechanical strength of repairs of the rotator cuff. J Bone Joint Surg $\mathrm{Br}$ 1994;76(03):371-380

67 Diop A, Maurel N, Chang VK, Kany J, Duranthon LD, Grimberg J. Tendon fixation in arthroscopic latissimus dorsi transfer for irreparable posterosuperior cuff tears: an in vitro biomechanical comparison of interference screw and suture anchors. Clin Biomech (Bristol, Avon) 2011;26(09):904-909

68 Goldstein Y, Grimberg J, Valenti P, Chechik O, Drexler M, Kany J. Arthroscopic fixation with a minimally invasive axillary approach for latissimus dorsi transfer using an endobutton in massive and irreparable postero-superior cuff tears. Int J Shoulder Surg 2013;7 (02):79-82

69 Aoki M, Okamura K, Fukushima S, Takahashi T, OginoT. Transfer of latissimus dorsi for irreparable rotator-cuff tears. J Bone Joint Surg Br 1996;78(05):761-766

70 Gerber C, Maquieira G, Espinosa N. Latissimus dorsi transfer for the treatment of irreparable rotator cuff tears. J Bone Joint Surg Am 2006;88(01):113-120

71 Elhassan BT, Wagner ER, Werthel JD. Outcome of lower trapezius transfer to reconstruct massive irreparable posterior-superior rotator cuff tear. J Shoulder Elbow Surg 2016;25(08): 1346-1353

72 Reddy A, Gulotta LV, Chen X, et al. Biomechanics of lower trapezius and latissimus dorsi transfers in rotator cuff-deficient shoulders. J Shoulder Elbow Surg 2019;28(07):1257-1264

73 Elhassan BT, Alentorn-Geli E, Assenmacher AT, Wagner ER. Arthroscopic-Assisted Lower Trapezius Tendon Transfer for Massive Irreparable Posterior-Superior Rotator Cuff Tears: Surgical Technique. Arthrosc Tech 2016;5(05):e981-e988

74 Valenti P, Werthel JD. Lower trapezius transfer with semitendinosus tendon augmentation: Indication, technique, results. Obere Extrem 2018;13(04):261-268 